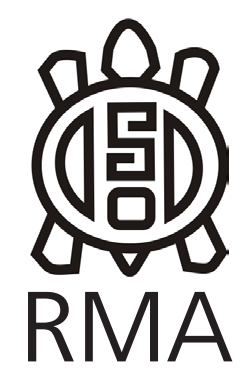

VI Jornadas

Arqueológicas Cuyanas

\title{
Investigaciones arqueológicas en el sitio Alero Dupuy, provincia de San Luis
}

\author{
Archaeological research \\ at Alero Dupuy site, San Luis province
}

\author{
Rafael P. Curtoni*, Gustavo Gómez ${ }^{* *}$, \\ Mariángeles Borgo***, Jorge Chiesa**** Verónica Lalinde*, \\ Víctor Martínez* y Augusto Oliván*****
}

*UE INCUAPA-CONICET, Facultad de Ciencias Sociales, Universidad Nacional del Centro de la provincia de Buenos Aires, Olavarría, Buenos Aires. E-mail: rcurtoni@soc.unicen.edu.ar **UE INCUAPA-Facultad de Ciencias Sociales, Universidad Nacional del Centro de la provincia de Buenos Aires, Olavarría, Buenos Aires. E-mail: ggomez@soc.unicen.edu.ar ${ }^{* * *}$ CONICET, Departamento de Geología, Facultad de Ciencias Físico-Matemáticas y Naturales, Universidad Nacional de San Luis. E-mail: mariangeles_borgo@hotmail.com ****JDepartamento de Geología, Facultad de Ciencias Físico-Matemáticas y Naturales, Universidad Nacional de San Luis. E-mail: jchiesa@unsl.edu.ar

*UE INCUAPA-CONICET, Facultad de Ciencias Sociales, Universidad Nacional del Centro de la provincia de Buenos Aires, Olavarría, Buenos Aires. E-mail: verolali@gmail.com

*UE INCUAPA-CONICET, Facultad de Ciencias Sociales, Universidad Nacional del Centro de la provincia de Buenos Aires, Olavarría, Buenos Aires. E-mail: victormq85@gmail.com *****Facultad de Ciencias Sociales, Universidad Nacional del Centro de la provincia de Buenos Aires, Olavarría, Buenos Aires.

\section{Resumen}

En esta nota se presentan los resultados obtenidos de las investigaciones arqueológicas desarrolladas en el sitio Alero Dupuy, departamento de Coronel Pringles, provincia de San Luis. Los materiales recuperados en las excavaciones permiten plantear la realización de actividades múltiples por parte de grupos cazadores recolectores posiblemente con ocupaciones redundantes desde el Holoceno medio al tardío.

Palabras clave: arqueología; Alero Dupuy; San Luis.

\section{Abstract}

This paper presents the results of archaeological research carried out on the site Alero Dupuy, Coronel Pringles, at San Luis province. The material recovered from excavations allows proposing that multiple activities were carried out by hunters and gatherers with redundant occupations from middle to late Holocene.

Keywords: archaeology; Alero Dupuy; Rockshelter, San Luis.

El objetivo de esta nota es dar a conocer los primeros resultados de las investigaciones arqueológicas desarrolladas en la Localidad arqueológica Dupuy, ubicada en cercanías de la ciudad de La Toma, provincia de San Luis. Los diversos sitios que componen esta localidad se encuentran en el sector centro-norte del área de estudio la cual comprende la cuenca media y superior del Río Quinto, incluyendo parte de las Sierras de San Luis y las estribaciones de Yulco-San José del Morro con las llanuras adyacentes (Figura 1). La vegetación predominante en el área es la del espinal, sobresaliendo las isletas de caldenes, algarrobos y chañares en las cercanías de los cauces de agua y los pastizales de llanuras (Cabrera y Willink 1980; Rosa et al. 2000).

En ese contexto interesa conocer las dinámicas de poblamiento en relación a los ríos que circundan el área (e.g. Río Quinto, Río Grande, Río Cañada Honda, Río Rosario, Río Conlara), y arroyos menores relacionados a la estructura hidrográfica, dado que conectan espacios

Recibido 11-03-2016. Recibido con correcciones 10-09-2016. Aceptado 04-11-2016

Revista del Museo de Antropología, Suplemento Especial 1: 43-50, 2017 / ISSN 1852-060X (impreso) / ISSN 1852-4826 (electrónico) 


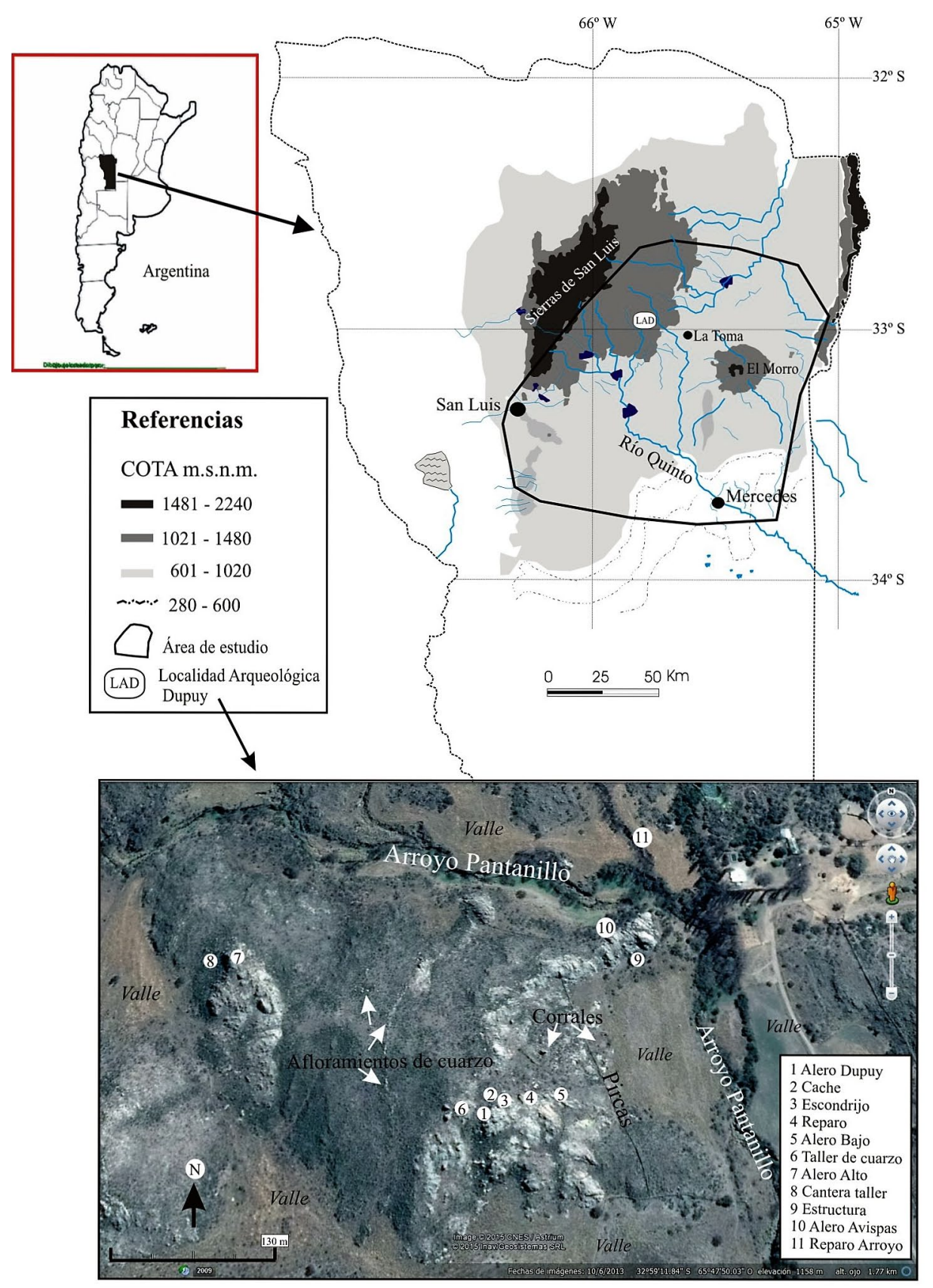

Figura 1: Localización del área de estudio y detalle de la Localidad Arqueológica Dupuy, provincia de San Luis.

Figure 1: Location of the study area in the province of San Luis and detail of the archaeological Dupuy locality.

Centrales como del ecosistema de llanuras de la Región Pampeana. Algunos de los propósitos planteados a mediano y largo plazo se vinculan con analizar la relación de los asentamientos arqueológicos con las topografías y geoformas del paisaje en las cuales se encuentran los mismos; caracterizar la organización tecnológica de los contextos arqueológicos recuperados; estimar aspectos vinculados a la subsistencia y dieta de los grupos humanos que ocuparon el área, y generar información cronológica de base que permita contextualizar temporalmente las ocupaciones humanas, entre otros. Los trabajos de campo realizados hasta el momento permitieron identificar más de 20 sitios arqueológicos localizados en pequeños abrigos rocosos, aleros y en menor medida a cielo abierto. En esta oportunidad presentamos algunas observaciones preliminares de los principales hallazgos

de las sierras con valles y planicies abiertas. De esa forma, se plantea el supuesto que el poblamiento inicial del área de estudio, posiblemente en el Holoceno temprano, se habría dado en los sectores de los valles interserranos, en lugares bajos y protegidos, por donde surcan ríos y arroyos pequeños y medianos semipermanentes, los cuales habrían actuado como vectores en los procesos de ocupaciones del paisaje. Estas pequeñas redes hidrográficas pudieron haber actuado como concentradores de recursos de subsistencia, siendo importantes en épocas de mayor aridez, y también como conectores del paisaje uniendo diferentes espacios.

La escala espacial seleccionada representa un ambiente de ecotono donde confluyen geoformas y condiciones ecológicas tanto del sistema serrano de las Sierras recuperados en el sitio Alero Dupuy y las cronologías absolutas obtenidas hasta el momento.

\section{Localidad Arqueológica Dupuy}

Los sitios que componen esta Localidad arqueológica se localizan a unos $15 \mathrm{~km}$ al noroeste de la ciudad de La Toma en un sector de sierras bajas, pequeñas quebradas, valles y mesetas. El contexto geomorfológico corresponde a las estribaciones meridionales de las Sierras de San Luis, con afloramientos de rocas ígneas y metamórficas del basamento Paleozoico. En el área de estudio, el basamento también comprende efusiones volcánicas, de mármol y calcretes del Neógeno, con una activa meteorización físico-química que permite la formación de cuevas y abrigos rocosos. En el mismo contexto de 


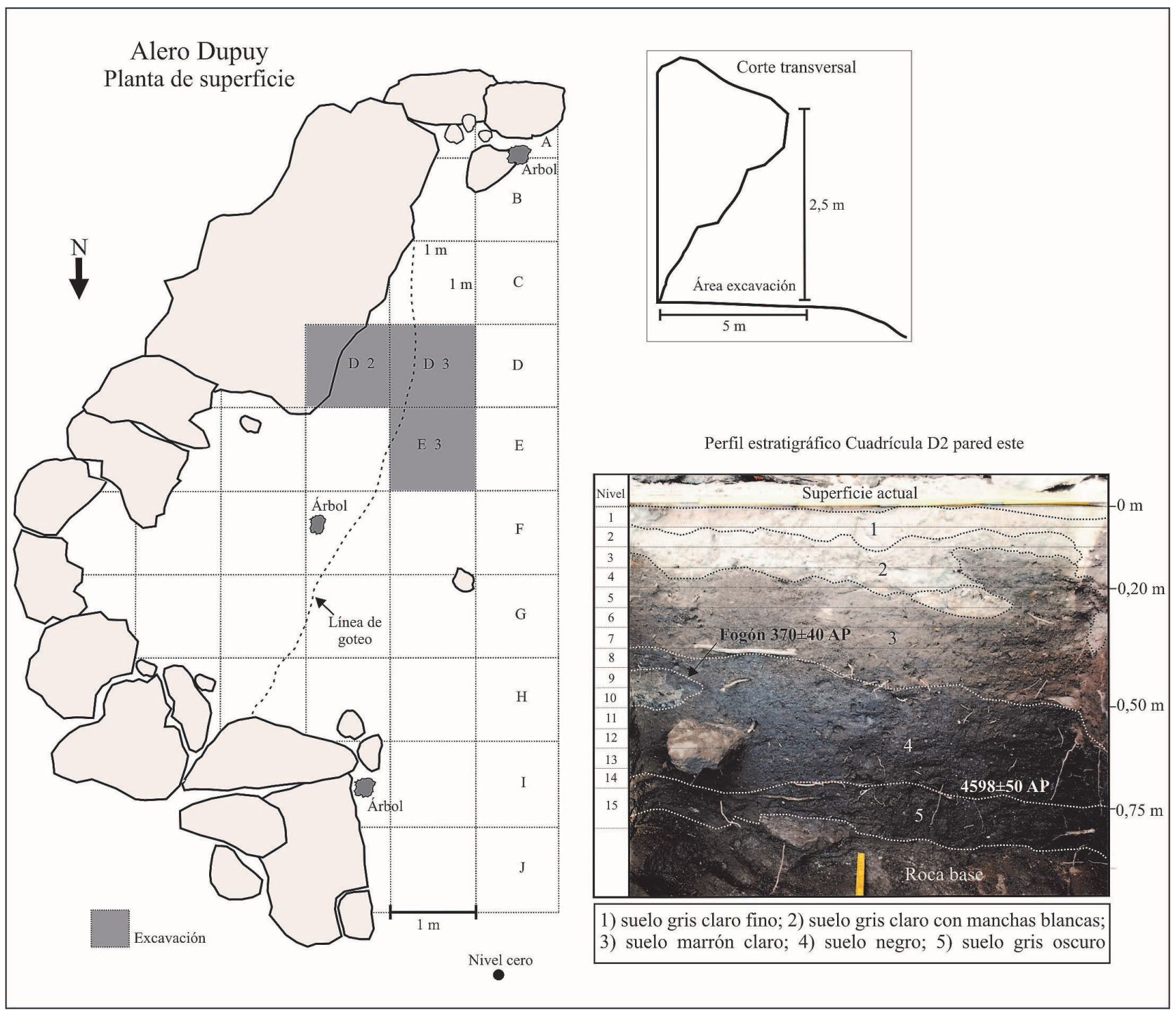

Figura 2: Planta de superficie del sitio Alero Dupuy y perfil estratigráfico.

Figure 2: Surface area of Alero Dupuy site and stratigraphic profile.

erosión se formaron pequeñas depresiones o pampas altas que luego fueron cubiertas por sedimentos eólicos y aluviales del Cuaternario (Di Paola 1994).

La Localidad arqueológica Dupuy está conformada por el sitio Alero Dupuy; un posible "cache" realizado en un pequeño "tafoni" cerrado con pirca seca; un arreglo de rocas tipo escondrijo; un reparo rocoso con material lítico en superficie; un pequeño alero con escasas evidencias en superficie (Alero Bajo); un taller de cuarzo ubicado enfrente de Alero Dupuy; un alero cercano con abundante material lítico en superficie (Alero Alto); una cantera taller de cuarzo; una estructura rectangular de pirca con montículo en el centro y ubicado en la cabecera del valle; un pequeño alero con escaso material arqueológico en superficie (Alero Avispas); un sitio a cielo abierto con material lítico y de molienda en superficie (Reparo del Arroyo), y una serie de corrales y pircas de diferentes tamaños (ver Figura 1). Topográficamente la
Localidad arqueológica Dupuy se encuentra limitada por el Arroyo Pantanillo al norte y al este y por pequeños valles al sur y al oeste. En un espacio aproximado de $350 \mathrm{~m}$ por $300 \mathrm{~m}$ se encuentran diferentes sitios, rasgos arqueológicos y afloramientos de cuarzo. El sitio Alero

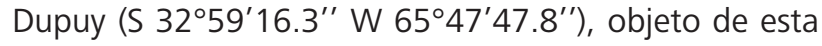
comunicación, tiene unos 8 metros de frente por 5 metros de fondo, con una recamara interna y un sector derrumbado. En el sector externo relacionado a la línea de goteo del alero se excavaron $3 \mathrm{~m}^{2}$ (Figura 2). La excavación se efectuó por medio de niveles artificiales de $5 \mathrm{~cm}$ combinada con el seguimiento y observación de la estratigrafía natural. Ello permitió identificar tres etapas geológicas de formación del depósito y cinco suelos o componentes sedimentarios distintos (ver más adelante). Las excavaciones realizadas permitieron recuperar numerosos instrumentos líticos, artefactos manufacturados por abrasión, picado y/o pulido y modificados por uso; abundante cantidad de desechos 
de talla, retalla y microretoque; diferentes instrumentos óseos; algunos tiestos de cerámica; numerosos restos óseos de diferentes especies; restos minerales, pigmentos y un pequeño fragmento de metal (posiblemente de cobre), en una columna estratigráfica de $0.80 \mathrm{~m}$ de potencia.

También se identificó una estructura de combustión con forma de cubeta de $30 \mathrm{~cm}$ de diámetro y $12 \mathrm{~cm}$ de espesor en la mitad de la secuencia (Niveles 9 y 10). Se efectuó un fechado radio-carbónico de espículas de carbón recuperadas en la estructura de combustión que arrojó una antigüedad de $370 \pm 40$ AP (LP 2878), el cual ubica a esta parte de la secuencia estratigráfica en momentos cercanos a las ocupaciones hispánicas. Es posible que la estructura de combustión haya sido cavada intencionalmente dado que pareciera representar un evento intrusivo en el suelo 4 cuya base fue datada en el Holoceno medio (ver más adelante). En la cuadrícula D3 y cerca del nivel de base de la excavación se registró una alineación de rocas que podría corresponder con un pircado de cierre del alero estando a su vez en relación con la actual línea de goteo del mismo.

En los niveles inferiores se recuperó un diente humano del cual se obtuvieron muestras de sarro para realizar análisis de fitolitos. Ello permitió identificar 16 fitolitos, de los cuales 5 corresponden a tricomas, 1 es un fragmento de espora y 8 pertenecen a tejidos de plantas. Uno de los fitolitos se corresponde con células de esclerénquima, siendo comunes en las familias de Chrysobalanaceae, Flacourtiaceae, Magnoliaceae, Moraceae, Fabaceae y Erythroxylaceae (Pearsall 2000, Piperno 2006). Existe una alta probabilidad que dicho fitolito pertenezca a la familia Fabaceae que es la más común en el área de estudio. Sin embargo, para poder identificarlo es necesario realizar comparaciones con muestras de la zona. Además, se recuperaron dos fitolitos alargados que pertenecen a hojas de la familia Poaceae, no pudiéndose por el momento obtener una identificación más específica. Por otra parte, de los últimos niveles de excavación (Nivel 14) se obtuvo otro fechado radio-carbónico por AMS sobre un fragmento óseo de guanaco con marcas de corte arrojando una antigüedad de 4,598 \pm 50 AP (AA 105423), ubicando a las primeras ocupaciones del alero hacia el Holoceno medio. Por el momento, las características del conjunto arqueológico recuperado no presentan diferencias significativas en su composición que permita plantear la presencia de componentes de ocupación distintos.

\section{Observaciones estratigráficas}

Los estudios geológicos realizados en los perfiles del Alero Dupuy indican la presencia de tres etapas en la conformación de los depósitos estratigráficos y cinco suelos o componentes sedimentarios distintos (ver Figura 2). La clasificación granulométrica y mineralógica de las muestras recogidas fue ejecutada en el Laboratorio de Sedimentos (Departamento de Geología, UMSL) utilizando la técnica de tamizado común y la metodología Bouyoucos. Desde la superficie actual hasta los $20 \mathrm{~cm}$ de profundidad se registra una disminución de grava, una proporción constante de arenas, un marcado aumento de limo y materia orgánica y al menos en el interior del alero el agua parece haber estado disponible para mantener la humedad. Esta etapa se relaciona con los primeros niveles de excavación (Niveles 1 a 4), donde se recuperó la mayor cantidad de evidencias arqueológicas. Asimismo en este bloque se encuentran los suelos 1 y 2 de escasa potencia y el inicio del suelo 3 (ver Figura 2). Entre 20 y $50 \mathrm{~cm}$ se registran proporciones constantes de materiales gruesos (grava), finos (limo) y materia orgánica con contenido significativo de arena, probablemente la arena fina y algo de arena media sean origen eólico, sobre todo considerando que el limo está presente y los valores de humedad no han cambiado. Esta etapa se corresponde con los niveles de excavación medios (Niveles 5 a 8) y donde se encuentra la mayor parte del suelo 3 y los inicios del suelo 4, caracterizado este último por su contenido orgánico y color negro. Entre 50 y $75 \mathrm{~cm}$ se observa una fuerte disminución de grava, un aumento sustancial de limo, una proporción constante de arenas y un ligero aumento de la materia orgánica retenida en los limos. Estos análisis permiten estimar que en la base de la secuencia estratigráfica pudo haber habido acciones de escurrimiento y fuertes vientos que contribuyeron a disminuir la humedad seguido por un período posterior de estabilidad ambiental. Esta última etapa se corresponde con los últimos niveles de excavación (9 a 15) y con la presencia de los suelos 4 y 5 (Figura 2). Estas observaciones permiten identificar una secuencia estratigráfica de ocupación humana del alero que, de acuerdo a los fechados obtenidos, se inicia en el Holoceno medio y se mantiene de manera continua hasta los momentos del contacto hispano indígena en la región.

\section{Tecnología lítica}

El análisis tecno-morfológico de los instrumentos líticos permite conocer diferentes aspectos vinculados con la organización tecnológica de los grupos humanos que ocuparon el área, reconociendo las etapas de elaboración de los instrumentos y las posibles actividades que pudieron haberse realizado. El conjunto lítico recuperado en el sitio está conformado por núcleos, desechos de talla, abundantes microlascas, filos naturales e instrumentos. En esta oportunidad se presentan los resultados obtenidos del análisis de los instrumentos. Los mismos fueron analizados de acuerdo a los lineamientos tipológicos propuestos por Aschero (1975, 1983), considerando materias primas, dimensiones absolutas y relativas, estado y fragmentación de los mismos, forma base, serie técnica, situación de los lascados, clase técnica y grupos y subgrupos tipológicos. Además, los instrumentos se agruparon en formales e informales siguiendo en parte los criterios de Andrefsky 
(1998). Se registran 32 instrumentos líticos que provienen de los 15 niveles estratigráficos, resultado de los trabajos de excavación. En los primeros cinco niveles se concentra la mayor cantidad de artefactos, disminuyendo en frecuencia en los niveles restantes.

La materia prima que predomina en el conjunto y sobre la cual se han confeccionado todos los instrumentos es el cuarzo. Teniendo en cuenta el uso casi exclusivo de cuarzo en Alero Dupuy, se planteó como uno de los objetivos el relevamiento e identificación de potenciales afloramientos rocosos o canteras de cuarzo que pudieran haber sido utilizados como locus de extracción de formas base para proveer a los sitios de la localidad. En este sentido, se prospectaron los alrededores de los sitios registrándose diferentes afloramientos de cuarzo en las inmediaciones de los mismos. En relación con el sitio Alero Dupuy, se registraron dos locus diferentes o canteras de cuarzos blancos compuestos por bloques pequeños y medianos el primero y por bloques grandes y fijos el segundo con evidencias de extracciones en los mismos. En todos los sectores de extracción de cuarzo se relevaron numerosas evidencias muebles e inmuebles de actividades de talla y desechos de los mismos. De esta forma, es posible plantear que el aprovisionamiento de cuarzo en Alero Dupuy ha sido local e inmediato, estando disponible en los alrededores del mismo.

El grupo tipológico mayormente representado son las puntas de proyectil triangulares (19), seguido por raspadores (7) (filo lateral corto, filo frontal corto) y puntas entre muescas (3) (lateral y frontal). Además se identifica una raedera (filo lateral largo), un cuchillo de filo natural con dorso formatizado y un artefacto no diferenciado de formatización sumaria (Tabla 1). En cuanto a los tamaños predominan los instrumentos pequeños y cortos, mientras que en los módulos prevalecen medianos normales, medianos grandes y muy grandes. En lo que respecta a las puntas de proyectil estas son en su totalidad pequeñas y apedunculadas aunque un gran número se encuentran fracturadas. Se presentan en todos los niveles estratigráficos pero las mayores frecuencias aparecen en los niveles superiores. Las formas base sobre las que se confeccionaron los instrumentos son en su mayoría lascas internas, distinguiendo en orden de frecuencia lascas angulares, de arista y planas. El cuchillo es el único instrumento que presenta como forma base una lasca secundaria. La serie técnica de mayor frecuencia es el retoque marginal seguido por lascados simples de formatización. Estos instrumentos han sido elaborados principalmente mediante lascados unifaciales directos. Por último, la clase técnica predominante es la reducción bifacial.

En general, el conjunto artefactual responde al desarrollo de actividades de procesamiento y consumo como también al mantenimiento y manufactura de instrumentos evidenciado por el gran número de microlascas que aparecen en el sitio. Los grupos tipológicos representados se asocian, en mayor parte, al procesamiento primario y secundario de recursos animales y vegetales. En lo que hace específicamente a las actividades de producción lítica, predomina la reducción sumaria de núcleos de cuarzo a partir de la cual se pudieron obtener lascas con filos naturales utilizables y formas base para la confección de útiles escasamente formatizados. Los artefactos recuperados se caracterizan por su baja formatización y presumiblemente fueron elaborados, usados y descartados en el sitio. Aunque se pueden diferenciar las puntas de proyectil por tener un mayor nivel de trabajo invertido relacionado con la talla y el retoque bifacial.

\section{El registro arqueofaunístico}

El análisis tafonómico tiene como objetivo evaluar la integridad del conjunto zooarqueológico y determinar cuáles especies fueron incorporadas al registro de manera natural y cuales por explotación humana. El material faunístico recuperado es abundante y presenta diversas condiciones, registrándose tanto restos quemados, fragmentados como también enteros y articulados. Se analizaron 2612 elementos, de los cuales

\begin{tabular}{lccc}
\hline \multirow{2}{*}{ Instrumental } & Grupo Tipológico & Subtotal & Total \\
\hline Extractivo/defensa & Puntas de Proyectil & 19 & 19 \\
\hline \multirow{2}{*}{ Consumo/procesamiento } & Runta entre muescas & 3 & \multirow{2}{*}{12} \\
\cline { 2 - 3 } & Raederas & 1 & \\
\cline { 2 - 3 } & $\begin{array}{c}\text { Cuchillo de filo natural } \\
\text { con dorso formatizado }\end{array}$ & 1 & \\
\hline No determinado & $\begin{array}{c}\text { Artefacto no } \\
\text { diferenciado de } \\
\text { formatización sumaria }\end{array}$ & 1 & \multirow{2}{*}{1} \\
\hline Total & & 32 & \\
\hline
\end{tabular}

Tabla 1: Instrumentos extractivo/ defensa versus consumo/procesamiento de Alero Dupuy.

Table 1: Extractive/defense versus consumption/processing tools from Alero Dupuy 
se contabilizaron 1870 elementos óseos determinables y 742 indeterminables, sin contar astillas de huesos. Se registra una importante variedad de especies entre las cuales se destacan Lama guanicoe, Ozotoceros bezoarticus, Zaedyus pichiy, Chaetophractus villosus, Ctenomys sp., Galea musteloides, como así también se han detectado restos que no han podido identificarse a nivel de especie pero si a categorías taxonómicas más inclusivas como cérvidos, armadillos, félidos, carnívoros, anuros, peces, aves. Además se han registrado, en menor medida, algunas especies introducidas como Bos taurus, Ovis aries, Eqqus cavallus y Lepus europaeus. Estos restos se encuentran en los niveles superiores de la excavación y en relación contextual con raíces de chañar las cuales podrían haber incidido en el transporte vertical y horizontal de los mismos.

Los elementos más abundantes corresponden a Lama guanicoe (NISP: 177), siendo las partes distales de las extremidades las más numerosas, junto con las costillas y las vértebras. En el caso de Ozotoceros bezoarticus los huesos del autopodio, vértebras y falanges son las más abundantes (NISP: 88). En lo que respecta a Lama guanicoe, se ha registrado un gran número de metapodios, escápulas y hemipelvis. En tanto Ozotoceros bezoarticus señala una mayor presencia de elementos craneales, tibias y elementos distales de las extremidades. Estas representaciones esqueletarias pueden estar relacionadas con el acarreo de ciertas partes del animal al sitio para su posterior procesamiento y consumo. El registro de partes esqueletarias pertenecientes a individuos jóvenes alcanza un 5,6\% del registro de elementos determinables como indeterminables. Se considera elementos juveniles a aquellos que no han soldado completamente sus partes esqueletarias, en especial aquellas partes articulares.

Los resultados parciales muestran un excelente estado de preservación, sin embargo, un alto porcentaje de huesos muestran fracturas irregulares que se producen cuando el hueso está seco, probablemente por factores diagenéticos tales como el pisoteo y la caída de rocas. Algunos elementos han sufrido la acción de raíces, que conlleva a la fracturación y al procesamiento de los nutrientes inorgánicos de los huesos por parte de la acción simbiótica de las raíces y los hongos. Las marcas de manganeso también han sido registradas en algunos restos, probablemente debido al estancamiento de agua en algunos sectores del sitio. Con respecto a la integridad del registro óseo, los factores que producen fracturas en los huesos pueden ser bioestratinómicos o bien diagenéticos. Los bioestratinómicos son aquellos que actúan antes del enterramiento inicial, como pueden ser la actividad de los cazadores, que en general producen fracturas helicoidales o espirales. En relación con esto último, es relevante el porcentaje de elementos óseos con huellas antrópicas (4\%) y quemados (22\%), y la presencia de lascas e instrumentos óseos (punzones, agujas, fragmentos de puntas, alisadores).

\section{Comentarios finales}

Las investigaciones arqueológicas en la provincia de San Luis tuvieron diferentes intensidades en su desarrollo y también períodos de discontinuidad en los cuales hubo ausencia de estudios sistemáticos y sostenidos (Heider y Curtoni 2016). En los últimos años se han realizado algunas investigaciones que remarcan la profundidad temporal de las ocupaciones humanas para la provincia, sobre todo a partir de los hallazgos arqueológicos realizados en Estancia La Suiza, en las Sierras de la Estanzuela, al sur de las Sierras de Comechingones, donde se recuperaron puntas colas de pescado en sitios a cielo abierto (Sario 2009, 2011; Laguens et al. 2007, 2009). En particular el área de estudio de este trabajo carece de investigaciones previas que permitan tener una primera caracterización y contextualización sobre su ocupación. En ese contexto, interesa generar nueva información arqueológica para discutir el poblamiento inicial del área a través de las vías de menor costo que toma como eje los ríos de llanura y la búsqueda de condiciones ambientales más favorables (Laguens et al. 2009, González 1960). Para momentos más tardíos, los trabajos de Gambier (1998) en las Sierras de San Luis permitieron reconocer algunos sitios con cerámica y cronologías cercanas a 1200 AP, constituyendo una referencia cronológica temprana para la alfarería de Sierras Centrales. En el piedemonte sur de las sierras de San Luis este investigador identificó algunos sitios a cielo abierto (como Sulupe), donde se registraron puntas de proyectil triangulares, manos de molino, restos de camélidos, armadillos y huevos de Rheidae, además de escasos fragmentos de cerámica. Por otra parte, el sitio Los Pedernales consiste de un abrigo rocoso localizado en la Sierra de San Luis, en un microambiente de altura donde el nivel de ocupación inicial fue datado en ca. 1200 AP (Gambier 1998). El contexto incluye fragmentos cerámicos, en este caso numerosos al igual que las puntas de proyectil triangulares. También se obtuvieron manos de molino y especímenes faunísticos asignados a camélidos, cérvidos y huevos de ñandú.

Para las áreas serranas de la provincia de Córdoba se cuentan con numerosos trabajos arqueológicos que dan cuenta de las diversidades de ocupaciones y de la amplitud temporal del poblamiento humano. En relación con las ocupaciones tardías, atribuidas a una etapa agroalfarera se han registrado varios sitios a cielo abierto en los fondos de valle, pudiéndose identificar dispersiones superficiales y sitios residenciales (Berberían 1984, Laguens 1999, Medina 2009, Pastor 2007). Las interpretaciones generadas a partir de los análisis intersitios de la región indican que se dieron importantes procesos de intensificación en el uso de la mayoría de los microambientes serranos y sus recursos, generándose numerosos sitios pequeños y en abrigos rocosos, los cuales tienden a concentrarse en las cabeceras de las quebradas de las sierras (Pastor et al. 2013, Rivero 2007). 
La información cronológica obtenida en Alero Dupuy señala que las ocupaciones humanas ocurrieron en momentos finales del Holoceno medio hasta el tardío pudiendo haber sido el lugar utilizado de manera recurrente como parte de las estrategias de movilidad y uso del paisaje por parte de grupos cazadores recolectores. El análisis arqueofaunístico preliminar permite delinear algunos aspectos de la subsistencia de las poblaciones prehispánicas e identificar la acción de diferentes agentes tafonómicos que intervinieron en la formación de los conjuntos óseos. La identificación anatómica y taxonómica de las muestras recuperadas plantea, en principio, la presencia de distintas estrategias de adquisición de recursos. En primer lugar, la caza de animales grandes y medianos como Lama sp. y Ozotoceros bezoarticus contribuyó para disponer, desde las ocupaciones iniciales hasta las finales de un recurso de subsistencia predecible y de alto rendimiento económico. El aprovechamiento de estos animales parece haber sido complementado con la adquisición de pequeños vertebrados, aves y la recolección de vegetales y huevos de Rheidae.

La diversificación e incremento en el manejo y explotación de distintos tipos de recursos, la incorporación de prácticas agrícolas de pequeña escala y el uso de todos los microambientes serranos es una característica macro-regional presente a partir del 1000 AP en Sierras Centrales (Laguens y Bonnin 2009; Pastor 2007; Rivero 2007; Medina 2009; López et al. 2014; Medina et al. 2014). Por el momento, en Alero Dupuy no se han hallado evidencias directas que sugieran la realización de prácticas agrícolas. Asimismo, no se registran cambios sustanciales en la composición del conjunto lítico y faunístico a lo largo de la secuencia estratigráfica que permita identificar cambios entre las ocupaciones iniciales y las finales. Por el contrario, se observa cierta continuidad en las tendencias de explotación de los recursos y en las estrategias tecnológicas implementadas. En síntesis, la información presentada permite plantear los inicios de ocupación del sitio por grupos cazadores recolectores hacia el Holoceno medio, posiblemente con estadías breves y posiblemente estacionales representadas por la menor densidad de evidencias registradas en los niveles inferiores. Por el contrario, en los niveles superiores el conjunto arqueofaunístico presenta una mayor densidad de restos así como también un aumento significativo del material lítico. Ello se correspondería con ocupaciones prolongadas y semipermanentes que se habrían dado hacia el Holoceno tardío. En consonancia con ello, los grupos humanos del área habrían desarrollado estrategias de ocupación intensiva del paisaje, aprovechando espacios y recursos tanto de las llanuras, los valles interserranos como de las sierras.

Olavarría, 19 de febrero de 2016

\section{Agradecimientos}

Las investigaciones se desarrollan gracias al apoyo de la UE INCUAPA-CONICET, Facultad de Ciencias Sociales, UNCPBA y del PICT 0290 de la Agencia Nacional de Promoción Científica y Tecnológica, Ministerio de Ciencia y Tecnología. Agradecemos a la familia Dupuy, Jorge, Viviana, Facundo, Pedro y Lucas, por el apoyo logístico en la provincia de San Luis. Al Ministerio de Turismo y las Culturas del Gobierno de la provincia de San Luis.

\section{Bibliografía}

Andrefsky, W. 1998. Lithics. Macroscopic approaches to analysis. Cambridge University Press. Cambridge.

Aschero, C. 1975. Ensayo para una clasificación morfológica de artefactos líticos aplicada a estudios tipológicos interpretativos. Informe inédito CONICET.

Aschero, C. 1983. Ensayo para una clasificación morfológica de artefactos líticos. Apéndices A y B. Apunte inédito para la Cátedra de Ergología y Tecnología, Facultad de Filosofía y Letras, Universidad de Buenos Aires, Buenos Aires.

Berberían, E. 1984. Potrero de Garay: Una entidad sociocultural tardía de la región serrana de la Provincia de Córdoba (Rep. Argentina). Comechingonia 4: 71-138.

Cabrera, A. L. \& Willink, W. 1980. Biogeografía de América Latina. Monografías Científicas de la Secretaría General de la Organización de los Estados Americanos, Programa Regional de Desarrollo Científico y Tecnológico, Washington D.C.

Di Paola, E. 1994. Distribución y evolución de los depósitos cenozoicos de la provincia de San Luis entre los $32^{\circ} 20^{\prime}$ y $34^{\circ}$ de latitud sur, Argentina. Revista Asociación Argentina Sedimentología, 1,33-52.

Gambier, M. 1998. Arqueología de la Sierra de San Luis. Instituto de Investigaciones Arqueológicas y Museo, Facultad de Filosofía, Humanidades y Artes, Universidad Nacional de San Juan, San Juan.

González, A. R. 1960. La estratigrafía de la gruta de Intihuasi (Prov. de San Luis, R.A.) y sus relaciones con otros sitios precerámicos de Sudamérica. Revista del Instituto de Antropología I: 5-296. Córdoba.

Heider G. \& R. Curtoni 2016. Investigaciones arqueológicas en la provincia de San Luis: a 150 años de sus inicios, historia y perspectivas. Ms.

Laguens, A. 1999. Arqueología del contacto hispanoindígena. Un estudio de cambios y continuidades en las Sierras Centrales de Argentina. BAR, International Series 801. Oxford, British Archaeological Reports. 
Laguens A., E. Pautassi, G. Sario \& R. Cattáneo 2007. Fishtail Projectil Points from Central Argentina. Current Research in Pleistocene 24: 55-57.

Laguens, A.\& M. Bonnin 2009. Sociedades indígenas de las Sierras Centrales. Editorial de la Universidad Nacional de Córdoba, Córdoba.

Laguens, A., R. Cattáneo, E. Pautassi \& G. Sario 2009. Poblamiento humano temprano en las Sierras de San Luis: Estancia La Suiza. En: Las sociedades de los paisajes áridos y semiáridos del centro-oeste argentino 1: 41-60. Río Cuarto.

López, M.L., M. Medina \& D. Rivero 2044. First records of Chenopodium spp./Amaranthus spp. starch grains and their relevance to the study of the late Holocene human subsistence in Central Argentina. The Holocene 1-8. Online version. DOI: 10.1177/0959683614558652.

Medina, M. E. 2009. Tendencias en el consumo prehispánico tardío de recursos faunísticos: Zooarqueología de C.Pun.39 y Puesto La Esquina 1 (Córdoba, Argentina). Archeofauna 18: $119-136$.

Medina, M., S. Pastor \& E. Berberían 2014. “Es gente fazil de moverse de una parte a otra". Diversidad en las estrategias de subsistencia y movilidad prehispánicas tardías (Sierras de Córdoba, Argentina). Complutum 25 (1): 73-88.

Pastor, S. 2007. Arqueología del Valle de Salsacate y pampas de altura adyacentes (Sierras Centrales de Argentina). Una aproximación a los procesos sociales del período prehispánico tardío (900-1573 d.C.). Tesis doctoral inédita. FCNyM, Universidad Nacional de La Plata, La Plata.
Pastor, S; M. Medina \& E. Berberián 2013. Poblados, Casas y Maizales. Arqueología de los Sitios Residenciales Tardíos de las Sierras de Córdoba (ca.1100-300 AP), Argentina Central. Revista Española de Antropología Americana 43: 31-55.

Pearsall, D., 2000. Paleoethnobotany: a Handbook of Procedures, second ed. Academic Press, San Diego.

Piperno, D., 2006. Identifying Manioc (Manihot esculenta Crantz) and other crops in Pre-Columbian Tropical America through starch grain analysis a case study from Central Panama. En: Zeder, M.A., Bradley, D.G., Emshwiller, E., Smith, B.D. (Eds.), Documenting Domestication New Genetic and Archaeological Paradigms. University of California Press, Berkeley, pp. 46e67.

Rivero, D. 2007. Ecología de los cazadores-recolectores en las Sierras de Córdoba. Tesis de Doctorado inédita. Facultad de Filosofía y Humanidades, Universidad Nacional de Córdoba, Córdoba.

Rosa, E. B., E. Scappini \& C. A. Bianco 2000. Gramíneas de la Sierra del Morro. Provincia de San Luis (Argentina). Universidad Nacional de San Luis, San Luis.

Sario, G. 2009. Estancia La Suiza 3 (provincia de San Luis): un estudio de la tecnología lítica. En: La zaranda de ideas, Revista de Jóvenes Investigadores en arqueología 5: 45-64. Buenos Aires.

Sario, G. 2011. Poblamiento humano en la provincia de San Luis: una perspectiva arqueológica a través del caso de la organización de la tecnología en Estancia La Suiza. Tesis doctoral Inédita. FFyH, Universidad Nacional de Córdoba. Córdoba. 